\title{
A double-blind, randomized, placebo- controlled trial of a computer-based Interpretation Bias Training for youth with severe irritability: a study protocol
}

Simone P. Haller ${ }^{1 *}$, Joel Stoddard ${ }^{2}$, Caroline MacGillivray ${ }^{3}$, Kelsey Stiles ${ }^{4}$, Gretchen Perhamus ${ }^{1}$, lan S. Penton-Voak ${ }^{5}$, Yair Bar-Haim ${ }^{6,7}$, Marcus R. Munafó ${ }^{5,8,9}$ and Melissa A. Brotman ${ }^{1}$

\begin{abstract}
Background: Severe, chronic, and impairing irritability is a common presenting clinical problem in youth. Indeed, it was recently operationalized as disruptive mood dysregulation disorder (DMDD) in the DSM-5. However, to date, there are no evidence-based treatments that were specifically developed for DMDD. The current randomized controlled trial assesses the efficacy of a computer-based cognitive training intervention (Interpretation Bias Training; IBT) in youth with DMDD. IBT aims to reduce irritability by altering judgments of ambiguous face-emotions through computerized feedback. IBT is based on previous findings that youth with irritability-related psychopathology rate ambiguous faces as more hostile and fear producing.

Methods/design: This is a double-blind, randomized controlled trial of IBT in 40 youth with DMDD. Participants will be randomized to receive four IBT sessions (Active vs. Sham training) over 4 days. Active IBT provides computerized feedback to change ambiguous face-emotion interpretations towards happy interpretations. Face-emotion judgments are performed pre and post training, and for 2 weeks following training. Blinded clinicians will conduct weekly clinical ratings. Primary outcome measures assess changes in irritability using the clinician-rated Affective Reactivity Index (ARI) and Clinical Global Impressions-Improvement (CGI-I) scale for DMDD, as well as parent and child reports of irritability using the ARI. Secondary outcome measures include clinician ratings of depression, anxiety, and overall impairment. In addition, parent and child self-report measures of depression, anxiety, anger, social status, and aggression will be collected.
\end{abstract}

Discussion: The study described in this protocol will perform the first RCT testing the efficacy of IBT in reducing irritability in youth with DMDD. Developing non-pharmacological treatment options for youth suffering from severe, chronic irritability is important to potentially augment existing treatments.

Trial registration: ClinicalTrials.gov, ID: NCT02531893. Registered on 25 August 2015.

Keywords: Irritability, Children and adolescence, Cognitive training, Interpretation bias, Randomized controlled trial, RCT, DMDD, Face-emotion

\footnotetext{
* Correspondence: simone.haller@nih.gov

${ }^{1}$ Section on Mood Dysregulation and Neuroscience, Emotion and

Development Branch, National Institute of Mental Health, 9000 Rockville Pike,

Building 15K, Bethesda, MD 20892, USA

Full list of author information is available at the end of the article
}

(c) The Author(s). 2018 Open Access This article is distributed under the terms of the Creative Commons Attribution 4.0 International License (http://creativecommons.org/licenses/by/4.0/), which permits unrestricted use, distribution, and reproduction in any medium, provided you give appropriate credit to the original author(s) and the source, provide a link to the Creative Commons license, and indicate if changes were made. The Creative Commons Public Domain Dedication waiver (http://creativecommons.org/publicdomain/zero/1.0/) applies to the data made available in this article, unless otherwise stated. 


\section{Background}

Irritability, defined as an increased proneness to anger and outbursts relative to peers $[1,2]$, is highly prevalent [3-7] and impairing in youth [8]. Irritability in youth predicts the development of depression and anxiety [3, $4,7]$, suicidality $[9,10]$, and is linked to overall reduced academic and socioeconomic functioning [11]. Given the functional impairment and association with negative outcomes, chronic irritability is a significant public health concern $[3-5,11,12]$.

Despite this pressing clinical and public health need, there is remarkably little treatment research on pathological irritablity $[1,2,13,14]$. Recently, disruptive mood dysregulation disorder (DMDD), with a core feature of chronic irritability, has been introduced into the Diagnostic and Statistical Manual of Mental Disorders 5 (DSM-5 [15]). DMDD provides a diagnostic category for trials of treatments targeting severe and impairing irritability in youth that may be readily translated into clinical practice. Currently, non-pharmacological treatment strategies for DMDD are investigational, with no evidence-based cognitive trainings to treat the irritability characteristic of DMDD.

\section{Rational for treatment intervention}

Clinically, irritability is characterized by a low threshold for angry, reactively aggressive responses to provocations [14]. One of the most established findings in youth with irritability-related psychopathology is that they are more likely to judge ambiguous social stimuli as hostile [14, 16]. Compared to healthy youth, chronically irritable youth preferentially attend towards threatening (i.e., angry) faces [17], and rate ambiguous faces as fear-inducing [18] or threatening (i.e., interpretation bias; [19]). A substantial body of work has shown that these biased interpretations of ambiguous social stimuli elicit and potentiate angry, aggressive responses in youth $[16,20]$. Hence, biases in interpreting social stimuli may serve to maintain irritability $[14,19]$ and provide a potential target for novel interventions.

One promising set of novel interventions relies on computer-based technology to change biases in information processing through repeated exposure to "corrective" feedback (e.g., [21]). This approach has shown promise in the treatment of anxiety (e.g., [22]). In the study described in this protocol, we will test whether such an approach can be used to shift biased interpretations of face-emotions in DMDD and thereby reduce irritability.

The Interpretation Bias Training (IBT) task [23] is a method to both quantify and train face-emotion interpretation bias. In this task, participants are presented with face-emotions morphed from very happy to very angry across a continuum of 15 stimuli (see Fig. 2 for example stimuli). Participants are instructed to make quick, binary happy-angry judgments for each face morph that appears on the screen. Morphs are presented in random order. Data indicate that both healthy participants and psychiatric patients categorize each morph reliably [23]. The morph in the continuum at which each participant's judgment switches from happy to angry has been termed "balance point" (BP) and represents a measure of interpretation bias. For instance, participants who rate only a few faces along the happy-angry continuum as happy before switching their judgment to predominantly angry responses would have a low BP, i.e., an increased bias towards perceiving ambiguous faces as angry. This IBT task can also be used to change face-emotion interpretations by providing trial-by-trial, computer-based feedback to shift the participant's BP along the continuum of face morphs.

In other phenotypes in youth, such as disruptive behavioral problems, preliminary data suggest that shifting participants' BP may reduce anger and aggression [24]. In a randomized controlled trial (RCT) of 46 adolescents who were in residential treatment because of aggressive behavior and high-risk of criminal behavior, Penton-Voak et al. [24] reported that four sessions of Active compared to Sham training on the IBT task shifted participants' BP toward happy and away from angry judgments. Further, Active training was associated with a significant reduction in both self-rated anger and aggressive behavior rated by blinded residential treatment staff.

In a recent preliminary study, Stoddard et al. [19] extended this work to youth with DMDD. In a series of pilot experiments, Stoddard and colleagues demonstrated a difference in BP between DMDD $(n=63)$ and healthy youth $(n=26)$, with DMDD youth exhibiting a bias towards angry judgments. Additionally, Stoddard and colleagues tested the feasibility of training irritable youth in an open pilot trial. In 14 DMDD youth, four sessions of Active training resulted in a shift in BP, which persisted for 2 weeks post training. Clinical data were consistent with an improvement in parent-reported irritability over the course of the open pilot trial. While promising, these data are preliminary and do not test efficacy, supporting a test of IBT on irritability in DMDD in a RCT. Thus, the objective of the current study, described in this protocol, is to assess the efficacy of IBT in reducing irritability in youth with DMDD in a double-blind, randomized, placebo-controlled design. The IBT RCT compares Active training, which encourages a shift in face-emotion interpretations toward happy judgments, to Sham training, where feedback does not encourage change in a person's pre-training face-emotion interpretations.

\section{Methods/design \\ Design}

The study described in this protocol performs a double-blind RCT to test the efficacy of IBT as an 
augmentation to standard of clinical care (i.e., psychotropic medications and/or psychotherapy) for DMDD (see Fig. 1). Forty youth with current DMDD will be randomized (1:1) to receive four IBT sessions (Active vs. Sham, in a double-blind design) over 4 days followed by weekly mood ratings and BP assessments for 2 weeks. Primary outcome measures in this trial assess changes in irritability using the Clinician Affective Reactivity Index (Clinician ARI) and Clinical Global Impressions-Improvement for DMDD (CGI-I; [25]), as well as parent- and child-report of irritability (child- and parent-rated ARI; [26]). Additional file 1 provides the SPIRIT checklist for this trial.

\section{Recruitment}

Patients will be recruited nationwide through Institutional Review Board (IRB)-approved postcards, mailings, announcements in newsletters and bulletin boards or centers, and contacts with support groups and approved websites. Information leaflets may be posted electronically on websites such as National Institutes of Health $(\mathrm{NIH})$ or National Institute of Mental Health (NIMH) websites, advocacy support group websites (e.g., National Alliance on Mental Illness (NAMI), Children and Adults with Attention Deficit/Hyperactivity Disorder), publications' websites (e.g., Washington Parent). Researchers (MAB) also give presentations to the public and mental health professionals regarding our research and recruitment efforts for studies.

Informed consent and assent will be obtained from parents and youth, respectively, before any trial-specific procedures are performed. All participants will be advised that research is entirely voluntary, that they may withdraw participation at any time. Families will be paid for participation.

At screening and during enrollment of the IBT RCT, participants will be informed of, and agree to, maintain their current mental health treatment regimen 2 weeks prior to the start of the IBT RCT and through the second follow-up visit.

\section{Participants \\ Inclusion criteria}

Participants must be between the ages of 8-17 years and currently meet full DSM-5 diagnostic criteria for DMDD as assessed by a structured clinical interview (Kiddie-Schedule for Affective Disorders and Schizophrenia Present and Lifetime Version; K-SADS-PL; [27]), with an additional supplement to assess the presence of DMDD [28]. Additionally, based on record review and interviews with child and parent, the research team must agree that the child's response to their current treatment is no more than minimal as operationalized by a DMDD CGI-S (1-month version) score of 3 or more. Patients must be fluent in English; both parents and youth must provide written informed consent and assent, respectively.

\section{Exclusion criteria}

Participants will be excluded if the irritability symptoms are due to the direct physiological effects of a drug, or

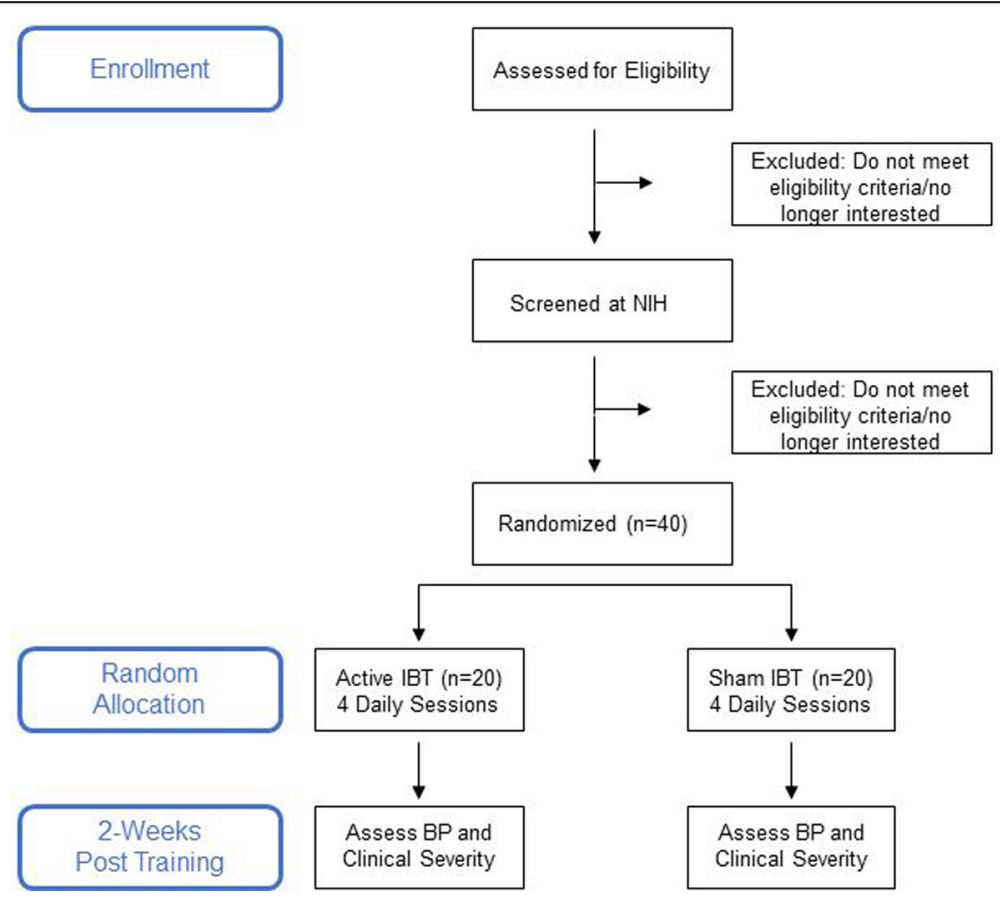

Fig. 1 Flow diagram of the Interpretation Bias Task randomized controlled trial (IBT RCT) 
to a general medical or neurological condition. Additionally, participants will not be eligible if they meet DSM 5-criteria, as assessed by the K-SADS-PL (Kaufman et al., 1997), for schizophrenia, schizophreniform disorder, schizoaffective illness, autism spectrum disorder, posttraumatic stress disorder, current major depressive disorder, or if they meet criteria for alcohol or substance abuse 3 months prior to enrollment. Participants will be excluded from the study if they exhibit cardinal bipolar symptoms or have an IQ below 70 as assessed by the Wechsler Abbreviated Scale of Intelligence (WASI; [29]). Patients must not have planned changes in outpatient psychiatric treatment regimen, which can include psychotropic medications and/or psychotherapeutic interventions, 2 weeks prior to enrollment and throughout the 3 weeks of the RCT training and post-training follow-up assessments.

\section{Intervention}

The IBT task assesses and trains happy-angry interpretations of face emotions. There are two versions of the task, an assessment version (Assessment Task) and a training version (Training Task, either Active or Sham). The task stimuli for both tasks consist of the same set of 15 face-emotion pictures, or "face morphs" (see Fig. 2 for example stimuli [30]). Face morphs were created from two prototypical happy and angry composite images of 20 individual male faces from the Karolinska Directed Emotional Faces [24, 30]. These happy and angry prototypical images were used as endpoints to generate a continuum of 15 images from unambiguously happy to unambiguously angry.

\section{Assessment Task}

In the Assessment Task, morphs are presented three times in random order for a total of 45 trials. Each trial consists of a fixation cross (1500-2500 ms), image presentation (150 ms), visual noise mask (250 ms), and a screen (no time limit) where a person is required to make a forced-choice response of "happy" or "angry" via keyboard button press. The Assessment Task takes approximately $2.5 \mathrm{~min}$. The Assessment Task measures the participant's BP, i.e., the morph at which the participant shifts their judgment from predominantly happy to predominately angry, with the continuum of morphs coded on an integer scale from the happiest endpoint $=1$ to the angriest endpoint $=15$. The BP can be estimated in real time during a training session as the proportion of happy responses to total responses multiplied by 15 .

\section{Training Task}

The Assessment Task is administered before and after each Training Task to measure BP pre and post each training session. In both the Active and the Sham training version of the task, the stimuli and timing are the same as the Assessment Task. The difference is that feedback is provided to the participant on every trial after their response. Feedback consists of a message saying "Right!/Wrong!" Consistent with previous IBT trials (e.g., [24]), in the Sham training, feedback is based on the participant's original $\mathrm{BP}$, as measured using the Assessment Task before training (i.e., the feedback is consistent with the participant's pre-training $\mathrm{BP}$ ). In the Active training, feedback is based on a modified version of the participant's pre-training BP. Specifically, the Active training is designed to shift the participant's BP so

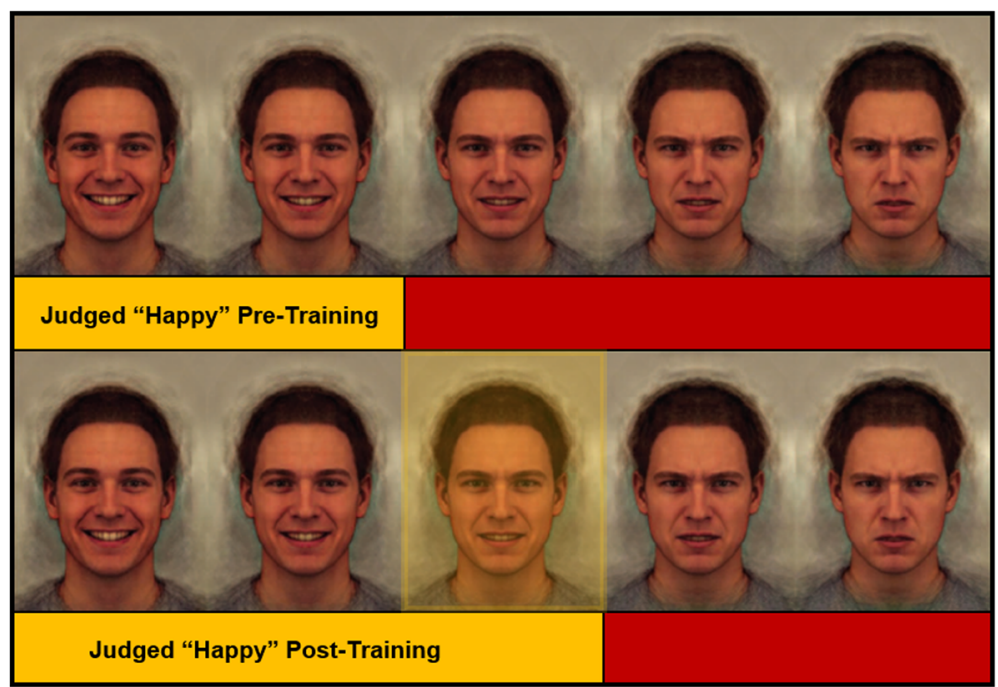

Fig. 2 Training is designed to shift pre-training balance point, thereby reducing an angry interpretation bias. Adapted with permission from the authors [22] 
that two ambiguous morphs that were rated angry at pre training will be rated as happy post training. The feedback is based on the participant's pre-training BP; hence two ambiguous images in the center of the morph continuum that the participant judged as angry pre training will instead be considered happy for the purposes of feedback. In the Training Task, each morph will be presented twice in random order to create a training block. There are six training blocks of 30 trials each summing to a total of 180 training trials.

\section{Outcome measures}

In addition to primary outcome measures assessing changes in irritability (ARI and CGI-I), this trial includes several secondary outcome measures. Secondary outcome measures include clinician ratings of depression, (Children's Depression Rating Scale, CDRS; [31]), anxiety (Pediatric Anxiety Rating Scale, PARS; [32]), and impairment (Clinical Global Impressions-Severity for DMDD, CGI-S; [25]; Children's Global Assessment Scale, CGAS; [33]). Additionally, parent-report measures of anxiety (Screen for Childhood Anxiety Related Emotional Disorders-parent, SCARED; [34]) and aggression (Modified Overt Aggression Scale, MOAS; [35]) and child self-report measures of depression (Children's Depression Inventory, CDI; [36]), anxiety (Screen for Childhood Anxiety Related Emotional Disorders-child, SCARED; [34]), anger (State-Trait Anger Expression Inventory-2 Child and Adolescent, STAXI-2 C/A; [37]). We will also collect information on social status (Kids in My Class Questionnaire, [38]; MacArthur Scale of Subjective Social Status; [39]) and intent attributions (Hostile Attribution Bias [HAB]) using a hypothetical-situations instrument [40].

\section{Study procedure}

Figure 1 provides a flow diagram of the study design, Fig. 3 provides a detailed schedule of enrollment, interventions and assessments. Participants will first complete a pre-training clinical screening before being enrolled in the protocol and randomized. Participants will be allocated to receive four IBT sessions (Active vs. Sham training in a double-blind design) over 4 days. Participants will complete a series of clinician- and self-report ratings pre and post training days followed by weekly clinical ratings and BP assessments for two additional weeks post training. The endpoint for primary outcome measures will be the DMDD CGI-I and ARI on the first follow-up visit (i.e., day 11). Figure 3 provides an overview of clinician-, parent-, and child self-report measures administered at the different time points. Participants' intervention allocation for the entire cohort will only be unblinded upon completion of the IBT study.

\section{Pre-training screening visit}

Participants and their parents will visit the NIH for a pre-training clinical assessment to determine eligibility to enroll in the IBT RCT. A trained masters- or doctoral-level clinician will conduct interviews with the participant and their parent including a current diagnostic interview (i.e., K-SADS-PL) and a clinical rating on the DMDD CGI-S.

\section{Training visits (days 1-4)}

Custom IBT software, written in Tcl (www.tcl.tk) (see also [19]), will be packaged with stimuli for Mac and PC computers. During visit day 1 , participants will complete the first session of IBT using either their own laptop computer or a NIH computer. All IBT data will be quality checked post training. Stimulus presentation timings will be inspected for each training session to ensure that timing of face displays is precise on non-NIH equipment. During this visit, participants and their parents are interviewed by clinicians for clinical ratings for irritability (cARI), depression CDRS), anxiety (PARS), overall functional impairment (CGAS), and severity of irritability and outbursts (CGI-S for DMDD, 1-week version). Participants and parents will also complete self-report forms. On day 2 and day 3, participants will complete one session of IBT, both sessions will be completed at home. The final training session on day 4 will again be onsite. On day 4 , the participant and their parent will again be interviewed by clinicians for clinical ratings for irritability (cARI), depression (CDRS), anxiety (PARS), overall functional impairment (i.e., CGAS), severity of irritability and outbursts (CGI-S for DMDD), and improvement (CGI-I for DMDD). The CGI-I interval will cover the time from day 1 through day 4, and the rating will be relative to the day-1 CGI-S. During this visit, participants and parents will again complete all self-report forms. To allow for reasonable flexibility, during days $1-4$, participants may reschedule up to two training sessions by extending the training period to day 5 or 6 to complete four once-daily sessions within a 6-day window. The participant completes rescheduled training sessions at home and self- and parent-report measures will be completed online. Clinical ratings may be done by phone.

\section{Follow-up visits \\ 1-week post training (day 11)}

This follow-up visit determines whether training effects persist for 1 week. Participants will begin this visit with an onsite Assessment Task to measure BP. Next, the participant and their parents will be interviewed for clinical ratings. The CGI-I interval is the past week, and the rating is relative to the day 1 CGI-S. During this visit, participants and parents will complete the same self-report measures. 


\begin{tabular}{|c|c|c|c|c|c|c|c|c|}
\hline \multirow{3}{*}{ TIMEPOINT } & \multicolumn{8}{|c|}{ STUDY PERIOD } \\
\hline & \multirow{2}{*}{$\begin{array}{c}\text { Enrollment } \\
-\mathrm{t}_{1}\end{array}$} & \multirow{2}{*}{$\frac{\text { Allocation }}{0}$} & \multicolumn{6}{|c|}{ Post-allocation } \\
\hline & & & $\begin{array}{c}\text { Day } \\
1\end{array}$ & $\begin{array}{c}\text { Day } \\
2\end{array}$ & $\begin{array}{c}\text { Day } \\
3\end{array}$ & $\begin{array}{c}\text { Day } \\
4\end{array}$ & $\begin{array}{c}\text { Day } \\
11\end{array}$ & $\begin{array}{c}\text { Day } \\
18\end{array}$ \\
\hline \multicolumn{9}{|l|}{ ENROLLMENT: } \\
\hline Eligibility screen & $\mathrm{x}$ & & & & & & & \\
\hline $\begin{array}{l}\text { Structured clinical interview (K- } \\
\text { SADS-PL) }\end{array}$ & $\mathrm{x}$ & & & & & & & \\
\hline $\begin{array}{l}\text { Wechsler Abbreviated Scale of } \\
\text { Intelligence (WASI) }\end{array}$ & $\mathrm{x}$ & & & & & & & \\
\hline $\begin{array}{l}\text { Clinical Global Impressions-Severity } \\
\text { (CGI-S) for DMDD (1-month version) }\end{array}$ & $\mathrm{x}$ & & & & & & & \\
\hline Informed consent & $\mathrm{x}$ & & & & & & & \\
\hline Allocation & & $\mathrm{x}$ & & & & & & \\
\hline \multicolumn{9}{|l|}{ INTERVENTIONS: } \\
\hline Active Training & & & $\mathrm{x}$ & $\mathrm{x}$ & $\mathrm{x}$ & $\mathrm{x}$ & & \\
\hline Sham Training & & & $\mathrm{x}$ & $\mathrm{x}$ & $\mathrm{x}$ & $\mathrm{x}$ & & \\
\hline \multicolumn{9}{|l|}{ ASSESSMENTS: } \\
\hline BP Assessment & & & $\mathrm{x}$ & $\mathrm{x}$ & $\mathrm{x}$ & $\mathrm{x}$ & $\mathrm{x}$ & $\mathrm{x}$ \\
\hline \multicolumn{9}{|l|}{ Clinician-rated measures } \\
\hline $\begin{array}{l}\text { Clinical Global Impressions-Severity } \\
\text { (CGI-S) for DMDD (1-week version) }\end{array}$ & & & $\mathrm{x}$ & & & $\mathrm{x}$ & $\mathrm{x}$ & $\mathrm{x}$ \\
\hline $\begin{array}{l}\text { Clinical Global Impressions- } \\
\text { Improvement (CGI-I) for DMDD }\end{array}$ & & & & & & $\mathrm{x}$ & $\mathrm{x}$ & $\mathrm{x}$ \\
\hline $\begin{array}{l}\text { Clinician Affective Reactivity Index } \\
\text { (ARI) }\end{array}$ & & & $\mathrm{x}$ & & & $\mathrm{x}$ & $\mathrm{x}$ & $\mathrm{x}$ \\
\hline $\begin{array}{l}\text { Children's Depression Rating Scale } \\
\text { (CDRS) }\end{array}$ & & & $\mathrm{x}$ & & & $\mathrm{x}$ & $\mathrm{x}$ & $\mathrm{x}$ \\
\hline $\begin{array}{l}\text { Pediatric Anxiety Rating Scale } \\
\text { (PARS) }\end{array}$ & & & $\mathrm{x}$ & & & $\mathrm{x}$ & $\mathrm{x}$ & $\mathrm{x}$ \\
\hline $\begin{array}{l}\text { Children's Global Assessment Scale } \\
\text { (CGAS) }\end{array}$ & & & $\mathrm{x}$ & & & $\mathrm{x}$ & $\mathrm{x}$ & $\mathrm{x}$ \\
\hline \multicolumn{9}{|l|}{ Parent-rated measures } \\
\hline $\begin{array}{l}\text { Screen for Childhood Anxiety Related } \\
\text { Emotional Disorders (SCARED) }\end{array}$ & & & $\mathrm{x}$ & & & $\mathrm{x}$ & $\mathrm{x}$ & $\mathrm{x}$ \\
\hline $\begin{array}{l}\text { Parent Affective Reactivity Index } \\
\text { (ARD) }\end{array}$ & & & $\mathrm{x}$ & & & $\mathrm{x}$ & $\mathrm{x}$ & $\mathrm{x}$ \\
\hline $\begin{array}{l}\text { Modified Overt Aggression Scale } \\
\text { (MOAS) }\end{array}$ & & & $\mathrm{x}$ & & & $\mathrm{x}$ & $\mathrm{x}$ & $\mathrm{x}$ \\
\hline \multicolumn{9}{|l|}{ Child-rated measures } \\
\hline $\begin{array}{l}\text { Screen for Childhood Anxiety Related } \\
\text { Emotional Disorders (SCARED) }\end{array}$ & & & $\mathrm{x}$ & & & $\mathrm{x}$ & $\mathrm{x}$ & $\mathrm{x}$ \\
\hline $\begin{array}{l}\text { Children's Depression Inventory } \\
\text { (CDI) }\end{array}$ & & & $\mathrm{x}$ & & & $\mathrm{x}$ & $\mathrm{x}$ & $\mathrm{x}$ \\
\hline $\begin{array}{l}\text { Child Affective Reactivity Index } \\
\text { (ARI) }\end{array}$ & & & $\mathrm{x}$ & & & $\mathrm{x}$ & $\mathrm{x}$ & $\mathrm{x}$ \\
\hline $\begin{array}{l}\text { State-Trait Anger Expression } \\
\text { Inventory-2 Child and Adolescent } \\
\text { (STAXI-2) }\end{array}$ & & & $\mathrm{x}$ & & & $\mathrm{x}$ & $\mathrm{x}$ & $\mathrm{x}$ \\
\hline Hostile Attribution Bias (HAB) & & & $\mathrm{x}$ & & & & & \\
\hline $\begin{array}{l}\text { Kids in My Class } \\
\text { Questionnaire }\end{array}$ & & & $\mathrm{x}$ & & & & & \\
\hline $\begin{array}{l}\text { MacArthur Scale of Subjective Social } \\
\text { Status }\end{array}$ & & & $\mathrm{x}$ & & & & & \\
\hline
\end{tabular}

Fig. 3 Schedule of enrollment, interventions, and assessments

\section{2-weeks post training (day 18)}

This follow-up is completed from home and determines whether training effects persist for 2 weeks. Participants will begin this session with an Assessment Task to measure BP. Next, the participant and their parent will be interviewed for clinical ratings. The CGI-I interval is the past week, and the rating is relative to the day-1 CGI-S. Participants and parents will complete self-report measures online.

\section{Allocation}

Intervention allocation sequence will be created via a computer-based random number generator. Randomization will be performed in blocks of 10 participants with a 1:1 ratio within blocks. Study personnel not involved in other aspects of the study will perform randomization. The document with the allocation sequence will be unavailable to those that enroll or otherwise interact with study participants. Training tasks will be prepared by study personal not otherwise 
involved in the study and IBT training tasks (Active vs. Sham) will be formatted identically. Trial participants, outcome assessors, data analysts, and research assistants interacting with families will be blind to participants' intervention allocation. The blind is not broken for the entire cohort until completion of the IBT RCT.

\section{Statistical analyses \\ Analysis of data/study outcomes}

Primary outcome measures are ARI and CGI-I. We will analyze both primary outcomes measures as a function of training group (Active vs. Sham) and time point (pre training and 1 week following training (day 11)). All data will be analyzed in accordance with the intention-to-treat principle. Following Stoddard et al. [19] and Pollak and Kistler [41], we will fit a logistic curve to the binary happy-angry judgment data to derive the BP. We will examine associations among BP, clinical ratings, and treatment condition. We expect any symptom changes in the Active training group to be associated with shifts in BP.

\section{Power analysis}

The open pilot trial [19] found a large effect of IBT on outcome ( $d=1.2$ for CGI-I). Because studies with similar computer-based approaches (see [42] for a meta-analysis) have found more moderate effect sizes in RCTs, we expect an effect size of $d=0.8$ to $d=1.0$. Power analyses suggest that 20 per group will be sufficient to detect differences at alpha $=0.05$, beta $=0.20$. Dropouts will be replaced for an $n=40$ of participants who completed the trial.

\section{Ethics}

Study procedures were approved by the National Institute of Mental Health (NIMH) Institutional Review Board (IRB). Risks associated with participation in this RCT are considered minimal. Adverse experiences include mild psychological distress and discomfort when responding to interview questions or completing questionnaires, or boredom during the IBT task. The principal and associate investigators will monitor patients closely throughout their participation in the RCT. After completing the RCT, all participants will be offered four Open Active IBT sessions, delivered on the same schedule as in the RCT and using the same primary outcome measures. For the Open Active IBT, while the principal and associate investigators will continue to monitor the patient, community providers are overseeing the patients' care.

Participants will be informed of their RCT intervention allocation upon completion of the trial.

An independent safety monitor will surveil data and safety. Audits of this treatment protocol will be conducted on a regular basis.

\section{Discussion}

This study is the first RCT of IBT in youth with DMDD. Chronic irritability, such as observed in DMDD, is severe and impairing for affected youth and their families, yet available treatment options are limited (Brotman et al., 2017). The inclusion of DMDD in the DSM-5 demonstrates the need to develop evidence-based treatments to target severe, pathological irritability. The current protocol describes the first double-blind RCT aimed to test the efficacy of IBT in reducing irritability in youth with DMDD. Forty youth are randomized to receive either four Active IBT or Sham IBT sessions in a double-blind design. Active IBT is designed to shift participants' pre-training BP along a face-morph continuum towards making increasingly happy judgments on ambiguous face morphs. Sham training does not change the participants' pre-training BP. BP is measured 2 weeks following treatment. Primary outcome measures assess changes in irritability pre to post training and 2 weeks after training using the clinician ARI and CGI-I for DMDD, as well as parent- and child-reports of irritability using the ARI. Secondary outcome measures include clinician ratings of depression, anxiety, and impairment. Parent- and child self-report measures of depression, anxiety, anger, social status, and aggression are also collected.

In this RCT, IBT is an augmentation to stable community treatment (pharmacological and/or psychosocial). Developing non-pharmacological treatment options for youth suffering from severe, chronic irritability is particularly important to minimize psychotropic medication exposure with profound side effects on the developing brain. Additionally, computer-based interventions have the potential to help bridge the treatment gap in psychological care as an easily accessible, cost-effective tool.

\section{Trial status}

The protocol (15-M-0182, Psychological Treatments for Youth with Severe Irritability) received final IRB approval in August 2015, after which trial recruitment commenced. Recruitment is expected to be completed by January 2019 .

\section{Limitations}

This study has several limitations. First, the sample size is modest to investigate potentially small therapeutic effects. Second, follow-up ratings will be conducted over 2 weeks following the intervention. Therefore, the study is limited in its ability to assess longer- term or delayed effects on symptoms. Third, we will not test near or far transfer of learning; face interpretations are trained and tested on the same face set. Should we find significant symptom reductions in the training group, examining whether training effects (i.e., changes in BP) generalize to new face stimuli would be a crucial next step to 
provide evidence for the mechanism by which active IBT may be efficacious. Lastly, we will only assess IBT as an add-on to stable community treatment. Participants enrolled in the trial will be concurrently taking a variety of different medications, including psychotropic medications. However, as discussed, we do require medications to remain stable throughout the trial.

\section{Additional file}

Additional file 1: SPIRIT 2013 Checklist: Study protocol for a double-blind, randomized, placebo-controlled trial of a computer-based Interpretation Bias Training for youth with severe irritability. (DOC $123 \mathrm{~kb}$ )

\begin{abstract}
Abbreviations
ARI: Affective Reactivity Index; BP: Balance point; CDI: Children's Depression Inventory; CGAS: Children's Global Assessment Scale; CGI-I: Clinical Global Impressions-Improvement for DMDD; CGI-S: Clinical Global Impressions Severity for DMDD; DMDD: Disruptive mood dysregulation disorder; DSM 5: Diagnostic and Statistical Manual of Mental Disorders 5; HAB: Hostile Attribution Bias; IBT: Interpretation Bias Training; IRB: Institutional Review Board; K-SADS: Kiddie-Schedule for Affective Disorders and Schizophrenia; MOAS: Modified Overt Aggression Scale; NIH: National Institutes of Health; NIMH: National Institute of Mental Health; PARS: Pediatric Anxiety Rating Scale; RCT: Randomized controlled trial; SCARED: Screen for Childhood Anxiety Related Emotional Disorders; STAXI-2 C/A: State-Trait Anger Expression Inventory-2 Child and Adolescent
\end{abstract}

\section{Acknowledgements}

The authors would like to thank Dr. Ellen Leibenluft and Dr. Daniel S. Pine for their valuable contributions and support throughout this study.

\section{Funding}

The authors' research is supported by the National Institute of Mental Health (NIMH) Intramural Research Program (ZIAMH002786-15, ZIAMH002778-17), conducted under NIH Clinical Study Protocols 15-M-0182 (ClinicalTrials.gov identifier: NCT02531893), 02-M-0021 (ClinicalTrials.gov identifier: NCT00025935), and 00-M-0198 (ClinicalTrials.gov identifier: NCT00006177). JS is supported by a career development award (K23MH1 13731).

\section{Availability of data and materials}

The datasets generated and/or analyzed during the current study are not currently publicly available. Data will be available in compliance with NIMH guidelines upon completion of the study.

\section{Authors' contributions}

$M B, J S, Y B H, I P V$, and $M M$ designed the study. MB, JS, SH, CM, KS, and GP implemented the design and/or oversee the data collection. SH and MB wrote the first draft of the manuscript. All authors contributed to and approved the final manuscript.

\section{Ethics approval and consent to participate}

Study procedures were approved by the National Institute of Mental Health Institutional Review Board. Informed consent and assent are obtained from parents and youth, respectively. Families are paid for participation.

\section{Consent for publication}

Not applicable.

\section{Competing interests}

The authors declare that they have no competing interests.

\section{Publisher's Note}

Springer Nature remains neutral with regard to jurisdictional claims in published maps and institutional affiliations.

\section{Author details}

${ }^{1}$ Section on Mood Dysregulation and Neuroscience, Emotion and Development Branch, National Institute of Mental Health, 9000 Rockville Pike, Building 15K, Bethesda, MD 20892, USA. ${ }^{2}$ Department of Psychiatry, University of Colorado School of Medicine, Aurora, USA. ${ }^{3}$ Lewis Katz School of Medicine, Temple University, Philadelphia, USA. ${ }^{4}$ Department of Psychology, University of California, Los Angeles, USA. ${ }^{5}$ School of Experimental Psychology, University of Bristol, Bristol, UK. ${ }^{6} \mathrm{~S} c h o o l$ of Psychological Sciences, Tel Aviv University, Tel Aviv, Israel. " Sagol School of Neuroscience, Tel Aviv University, Tel Aviv, Israel. ${ }^{8}$ MRC Integrative Epidemiology Unit, University of Bristol, Bristol, UK. ${ }^{9}$ UK Centre for Tobacco and Alcohol Studies, University of Bristol, Bristol, UK.

Received: 15 June 2018 Accepted: 3 October 2018

Published online: 14 November 2018

\section{References}

1. Brotman MA, Kircanski K, Leibenluft E. Irritability in children and adolescents. Annu Rev Clin Psychol. 2017a;13:317-41.

2. Brotman MA, Kircanski K, Stringaris A, Pine DS, Leibenluft E. Irritability in youths: a translational model. Am J Psychiatr. 2017b;174(6):520-32.

3. Brotman MA, Schmajuk M, Rich BA, Dickstein DP, Guyer AE, Costello EJ, Leibenluft E. Prevalence, clinical correlates, and longitudinal course of severe mood dysregulation in children. Biol Psychiatry. 2006a;60(9):991-7.

4. Brotman MA, Schmajuk M, Rich BA, Dickstein DP, Guyer AE, Costello EJ, et al. Prevalence, clinical correlates, and longitudinal course of severe mood dysregulation in children. Biol Psychiatry. 2006b;60(9):991-7.

5. Copeland WE, Shanahan L, Egger H, Angold A, Costello EJ. Adult diagnostic and functional outcomes of DSM-5 disruptive mood dysregulation disorder. Am J Psychiatry. 2014;171(6):668-74.

6. Copeland WE, Brotman MA, Costello EJ. Normative irritability in youth: developmental findings from the Great Smoky Mountains Study. J Am Acad Child Adolesc Psychiatry. 2015;54(8):635-42.

7. Vidal-Ribas P, Brotman MA, Valdivieso I, Leibenluft E, Stringaris A. The status of irritability in psychiatry: a conceptual and quantitative review. J Am Acad Child Adolesc Psychiatry. 2016;55(7):556-70.

8. Leibenluft E. Severe mood dysregulation, irritability, and the diagnostic boundaries of bipolar disorder in youths. Am J Psychiatry. 2011;168(2):129-42.

9. Pickles A, Aglan A, Collishaw S, Messer J, Rutter M, Maughan B. Predictors of suicidality across the life span: The Isle of Wight study. Psych Med. 2010; 40(09):1453-66.

10. Orri, M., Galera, C., Turecki, G., Forte, A., Renaud, J., Boivin, M., .Geoffroy, M. C. (2018). Association of childhood irritability and depressive/anxious mood profiles with adolescent suicidal ideation and attempts. JAMA Psychiatry 75(5): 465-473.

11. Stringaris A, Cohen P, Pine DS, Leibenluft E. Adult outcomes of youth irritability: a 20-year prospective community-based study. Am J Psychiatry. 2009:166(9):1048.

12. Stringaris A, Goodman R. Longitudinal outcome of youth oppositionality: irritable, headstrong, and hurtful behaviors have distinctive predictions. J Am Acad Child Adolesc Psychiatry. 2009;48(4):404-12.

13. Leibenluft E. Pediatric irritability: a systems neuroscience approach. Trends Cogn Sci. 2017;21(4):277-89.

14. Leibenluft E, Stoddard J. The developmental psychopathology of irritability. Dev Psychopathol. 2013;25(4 Pt 2):1473-87.

15. American Psychiatric Association. Diagnostic and Statistical Manual of Mental Disorders. 5th ed. Washington, DC: American Psychiatric Association; 2013.

16. Crick NR, Dodge KA. A review and reformulation of social informationprocessing mechanisms in children's social adjustment. Psychol Bull. 1994; 115(1):74-101.

17. Hommer, R. E., Meyer, A., Stoddard, J., Connolly, M. E., Mogg, K., Bradley, B. P., Brotman, M. A. (2014). Attention bias to threat faces in severe mood dysregulation. Depress Anxiety, 31(7), 559-565.

18. Brotman MA, Rich BA, Guyer AE, Lunsford JR, Horsey SE, Reising MM, Thomas LA, Fromm SJ, Towbin K, Pine DS, Leibenluft E. Amygdala activation during emotion processing of neutral faces in children with severe mood dysregulation versus ADHD or bipolar disorder. Am J Psychiatry. 2010;167(1):61-9.

19. Stoddard J, Sharif-Askary B, Harkins EA, Frank HR, Brotman MA, Penton-Voak IS, Leibenluft E. An open pilot study of training hostile interpretation bias to treat disruptive mood dysregulation disorder. J Child Adolesc PsychOpharmacol. 2016;26(1):49-57. 
20. Wilkowski BM, Robinson MD. The cognitive basis of trait anger and reactive aggression: an integrative analysis. Personal Soc Psychol Rev. 2008;12(1):3-21.

21. Bar-Haim Y, Lamy D, Pergamin L, Bakermans-Kranenburg MJ, Van ljzendoorn MH. Threat-related attentional bias in anxious and nonanxious individuals: a meta-analytic study. Psychol Bull. 2007;133(1):1.

22. Hakamata Y, Lissek S, Bar-Haim Y, Britton JC, Fox NA, Leibenluft E, et al. Attention bias modification treatment: a meta-analysis toward the establishment of novel treatment for anxiety. Biol Psychiatry. 2010;68(11):982-90.

23. Penton-Voak IS, Bate H, Lewis G, Munafo MR. Effects of emotion perception training on mood in undergraduate students: randomised controlled trial. $\mathrm{Br}$ J Psychiatry. 2012;201(1):71-2

24. Penton-Voak IS, Thomas J, Gage SH, McMurran M, McDonald S, Munafo MR. Increasing recognition of happiness in ambiguous facial expressions reduces anger and aggressive behavior. Psychol Sci. 2013;24(5):688-97.

25. Walkup JT, Labellarte MJ, Riddle MA, Pine DS, Greenhill L, Klein R, Klee B. Fluvoxamine for the treatment of anxiety disorders in children and adolescents. N Engl J Med. 2001;344(17):1279-85.

26. Stringaris A, Goodman R, Ferdinando S, Razdan V, Muhrer E, Leibenluft E, Brotman MA. The Affective Reactivity Index: a concise irritability scale for clinical and research settings. J Child Psychol Psychiatry. 2012;53(11):1109-17.

27. Kaufman J, Birmaher B, Brent D, Rao UMA, Flynn C, Moreci P, et al. Schedule for affective disorders and schizophrenia for school-age children-present and lifetime version (K-SADS-PL): initial reliability and validity data. J Am Acad Child Adol Psych. 1997;36(7):980-8.

28. Wiggins JL, Brotman MA, Adleman NE, Kim P, Oakes AH, Reynolds RC, Chen G, Pine DS, Leibenluft E. Neural Correlates of Irritability in Disruptive Mood Dysregulation and Bipolar Disorders. Am J Psychiatry. 2016;173(7):722-30.

29. Wechsler D. WASI Manual. San Antonio: Psychological Corporation; 1999.

30. Lundqvist D, Litton JE. The Averaged Karolinska Directed Emotional Faces AKDEF, CD ROM from Department of Clinical Neuroscience, Psychology section, Karolinska Institutet; 1998. isbn:91-630-7164-9.

31. Poznanski EO, Mokros HB. Children's Depression Rating Scale, Revised (CDRS-R). Los Angeles: Western Psychological Services; 1996.

32. Research Units on Pediatric Psychopharmacology Anxiety Study Group. The Pediatric Anxiety Rating Scale (PARS): development and psychometric properties. J Am Acad Child Adolesc Psychiatry. 2002;41(9):1061-9.

33. Shaffer D, Gould MS, Brasic J, Ambrosini P, Fisher P, Bird H, Aluwahlia S. A children's global assessment scale (CGAS). Arch Gen Psychiatry. 1983;40(11): 1228-31.

34. Birmaher B, Brent DA, Chiappetta L, Bridge J, Monga S, Baugher M. Psychometric properties of the Screen for Child Anxiety Related Emotional Disorders (SCARED): a replication study. J Am Acad Child Adolesc Psychiatry. 1999;38(10):1230-6.

35. Kay SR, Wolkenfeld F, Murrill LM. Profiles of aggression among psychiatric patients: I. Nature and prevalence. J Nerv Mental Dis. 1988;176(9):539-46.

36. Kovacs, M. (1992). Children's Depression Inventory: Manual. Multi-Health Systems.

37. Spielberger CD. STAXI-2: State-trait Anger Expression Inventory-2. Odessa: Psychological Assessment Resources; 1999.

38. Kochenderfer B, Ladd G. Peer victimization: cause or consequence of school maladjustment? Child Dev. 1996;67:1305-17.

39. Adler, N., Stewart, J. (2007). The MacArthur Scale of subjective social status. MacArthur Research Network on SES \& Health. Retrieved from http://www. macses.ucsf.edu/Research/Psychosocial/subjective.php.

40. Crick NR. Relational aggression: The role of intent attributions, feelings of distress and provocation type. DevPsych. 1995;7(02):313.

41. Pollak SD, Kistler DJ. Early experience is associated with the development of categorical representations for facial expressions of emotion. Proc Natl Acad Sci. 2002;99(13):9072-6.

42. Price RB, Wallace M, Kuckertz JM, Amir N, Graur S, Cummings L, Popa P, Calrbring P, Bar-Haim Y. Pooled patient-level meta-analysis of children and adults completing a computer-based anxiety intervention targeting attention bias. Clin Psychol Rev. 2016;50:37-49.

\section{Ready to submit your research? Choose BMC and benefit from:}

- fast, convenient online submission

- thorough peer review by experienced researchers in your field

- rapid publication on acceptance

- support for research data, including large and complex data types

- gold Open Access which fosters wider collaboration and increased citations

- maximum visibility for your research: over $100 \mathrm{M}$ website views per year

At BMC, research is always in progress.

Learn more biomedcentral.com/submissions 